Article

\title{
Efficacy of Drospirenone-Containing Hormone Replacement Therapy to Reduce Vasomotor Symptoms of Menopause
}

\author{
Dana A. Brown *, Amy Henneman and Darshana N. Desai \\ Lloyd L. Gregory School of Pharmacy, Palm Beach Atlantic University, 901 S. Flagler Drive, \\ PO Box 24708, West Palm Beach, FL 33416, USA; E-Mails: amy_henneman@pba.edu (A.H.); \\ Desaidn@pba.edu (D.N.D.)
}

* Author to whom correspondence should be addressed; E-Mail: dana_brown@pba.edu; Tel.: +1-561-803-2734; Fax: +1-561-803-2731.

Received: 28 June 2013; in revised form: 25 September 2013 / Accepted: 15 October 2013 / Published: 30 October 2013

\begin{abstract}
Hormone replacement therapy has been proven efficacious for controlling vasomotor symptoms such as hot flushes associated with menopause. Drospirenone is a progestin with antiandrogenic and antimineralocorticoid activity that may be used in combination with estrogen to control hot flushes and offers the potential benefit of minimizing breast tenderness, blood pressure elevations and weight gain. Six clinical trials were reviewed. Of these, four trials explicitly listed hot flushes as a primary outcome. Efficacy with regards to hot flushes was found to range from modest to large (i.e., $37.5 \%$ to $94.6 \%$ ), and four of the studies utilized diary cards to assess hot flushes. Results from these studies must be interpreted cautiously as quite a few limitations existed such as small population sizes involving specific ethnic groups, lack of $p$ values with regards to baseline characteristics lending question to homogeneity, and inclusion of mostly healthy participants. Additionally, while the studies were long enough to see an effect, the long term effects of drospirenone-containing hormone replacement therapy (HRT) is unknown. The available data supports the use of drospirenone-containing HRT for the treatment of hot flushes associated with menopause.
\end{abstract}

Keywords: drospirenone; estradiol-drospirenone combination; hormone replacement therapy; menopause; postmenopause; premenopause; climacteric; and vasomotor system 


\section{Introduction}

The association between vasomotor symptoms, including hot flushes (sometimes referred to as hot flashes) and night sweats, and menopause has long been recognized. Vasomotor symptoms occur in $50 \%$ to $85 \%$ of women, affecting approximately 360 million women over age 45 worldwide. Hot flushes occur most frequently and typically most intensely within the first two years after menopause, and are therefore most reported during this time. Women may experience hot flushes infrequently (i.e., weekly or monthly), daily, or even on an hourly basis. The reduction and overall change in hormones, particularly estrogen, associated with menopause appear to modulate the changes in temperature that occur in the hypothalamus, resulting in hot flushes [1].

Because vasomotor symptoms can negatively impact a woman's quality of life, women may decide to seek available options to diminish the frequency and severity of hot flushes. Hormone replacement therapy (HRT) has been proven efficacious for controlling these vasomotor symptoms as well as improving urogenital atrophy and decreasing osteoporosis development in women with menopause. Estrogen replacement remains the most effective option for the management of vasomotor symptoms. For women with an intact uterus, it is recommended that estrogen be combined with a progestin as a means of minimizing the risk for endometrial cancer [1-3].

Women may be hesitant to use HRT for vasomotor symptoms given the risks that are associated with therapy. In recent years, newer progestins have been developed with more favorable side effect profiles. Specifically, drospirenone, a derivative of $17 \alpha$-spironolactone, is a synthetic steroidal progestin possessing antimineralocorticoid and antiandrogenic activity. As compared with all other synthetic progestins, drospirenone resembles the pharmacological profile of endogenous progesterone. However, it is able to ultimately reduce sodium and water retention through blunting estrogen's ability to activate the renin-angiotensin-aldosterone system (RAAS). As such, adverse effects such as breast tenderness, increases in blood pressure and weight gain would be minimized [2-4]. Drospirenone's antiandrogenic properties minimize the untoward dermatological effects often associated with progestin use, such as hirsutism, acne and seborrhea [5,6].

Because of its effects on the RAAS, hyperkalemia is a concern with treatment. For this reason, it should not be used in women with adrenal insufficiency, renal impairment, or hepatic dysfunction. Additionally, caution should be used when prescribing drospirenone-containing HRT to women who are using medications that can increase potassium levels such as potassium-sparing diuretics, non-steroidal antiflammatory drugs, angiotensin-converting enzyme inhibitors or angiotensin receptor blockers. Additionally, similar to other estrogen-containing therapies, treatment with drospirenone-containing HRT should be avoided in women with venous thromboembolism (VTE) including a deep vein thrombosis and pulmonary embolism as well as estrogen-sensitive cancers. Lastly, risk factor assessment for cardiovascular disease should occur before starting therapy given the increased risk for myocardial infarction, stroke, and VTE with estrogen [7].

We conducted a literature search using MEDLINE to identify studies that assessed the effect of drospirenone in combination with estradiol for the treatment of vasomotor symptoms in postmenopausal women. The following terms were used in various combinations in the search strategy: drospirenone, estradiol-drospirenone combination, hormone replacement therapy, menopause, 
postmenopause, premenopause, climacteric, and vasomotor system. This article reviews the effects of drospirenone plus estradiol on vasomotor symptoms.

\section{Experimental Section}

Lee et al. [8] assessed the effects of estradiol $1 \mathrm{mg}$ /drospirenone $2 \mathrm{mg}$ on hot flushes and other climacteric symptoms in 90 postmenopausal Korean women. In a randomized, double-blind, placebo-controlled study, women ages 45 to 65 were randomized to receive estradiol/drospirenone $(n=45)$ or placebo $(n=45)$ for 16 weeks. They were required to experience at least five moderate to severe hot flushes on at least seven days of the two week pre-treatment period; have an intact uterus with no abnormal endometrial findings or have a hysterectomy; and be amenorrhic for at least one year, have a serum estradiol level $\leq 20 \mathrm{pg} / \mathrm{mL}$ and follicle stimulating hormone (FSH) level $\geq 50 \mathrm{U} / \mathrm{L}$ if amenorrhic for 6 to 12 months, have a hysterectomy at $<58$ years old with an estradiol level $\leq 20 \mathrm{pg} / \mathrm{mL}$ and FSH level $\geq 50 \mathrm{U} / \mathrm{L}$, or be $>58$ years of age with a hysterectomy. Patients were ineligible for study participation if they had abnormal cervical smear findings, undiagnosed genital bleeding, a myocardial infarction within six months of study participation, congestive heart failure, known or suspected malignancy, history of or current hepatic disease, uncontrolled thyroid condition, depression, stroke, diabetes, alcohol abuse, heavy smoker, hypertension, idiopathic thrombophlebitis, thromboembolic disease, adrenal insufficiency, severe renal impairment, pregnancy, lactation, or use of other hormone therapies within four weeks prior to study entry.

Participants were asked to take their study medications at the same time each day and to record daily symptoms and vaginal bleeding patterns on diary cards. The frequency and severity of the symptoms were recorded as mild (awareness of symptoms but tolerated), moderate (symptoms restricted but did not prevent daily activities), and severe (unable to perform daily activities). The primary outcome was the individual relative change in the mean number of hot flushes during weeks 3 through 16 as compared to baseline. Secondary outcomes included changes in climacteric symptoms such as sweating, insomnia, nervousness and depression as well as urogenital symptoms, vaginal bleeding, and assessment of serum levels of estradiol, estrone, and drospirenone.

The mean number of hot flushes per week was reduced by $84.4 \%$ in those receiving estradiol/drospirenone during weeks 3 to 16 compared to a $48.1 \%$ reduction in patients receiving placebo. When looking at severity, the frequency of moderate and severe hot flushes began to markedly decline after four weeks of treatment with estradiol/drospirenone. Other climacteric symptoms such as sweating, insomnia, depression, and nervousness were reduced with hormone therapy. Overall, 23 women experienced adverse effects during or after the study, with 14 of these women receiving active treatment and 9 receiving placebo. Breast pain and abdominal pain were the most common adverse effects. Other adverse effects included upper respiratory infection, surgery, dyspepsia, and chest pain.

The authors concluded that combination therapy with estradiol and drospirenone was safe and effective for treating hot flushes and other climacteric symptoms in postmenopausal Korean women. However, this study does have several limitations. The investigators comment that the distribution of the primary outcome was not normal and appropriately performed a non-parametric test on the data; however, all of the hot flush severities (mild, moderate, and severe) were analyzed in the primary outcome. This is perhaps why they did not have a normal distribution, and it is difficult to determine if 
one patient was experiencing all of the severe hot flushes, which could skew the data if numerous patients were experiencing them. Additionally, the primary outcome data was reported using a line graph with no numbers provided. The only numerical data given is the frequency of hot flushes per week according to severity. There was also a large placebo effect in this study, which lends question to the significance of the findings. Lastly, the external validity of this study is somewhat limited as this study occurred only in Korean women and included extensive exclusion criteria [8].

In a randomized, double-blind, placebo-controlled study, Schurmann et al. [9] assessed the efficacy of estradiol with various doses of drospirenone for the treatment of hot flushes in healthy menopausal women. Two hundred twenty five women ages 45 to 65 with at least five moderate to severe hot flushes occurring daily on at least 7 out of 14 days preceding the study were randomized to receive estradiol $1 \mathrm{mg}$ with drospirenone $1 \mathrm{mg}(\mathrm{n}=55), 2 \mathrm{mg}(\mathrm{n}=52)$ or $3 \mathrm{mg}(\mathrm{n}=57)$ or placebo $(\mathrm{n}=61)$ for 16 weeks. Eligible participants were required to have an intact uterus with normal endometrial findings, an estradiol level of $\leq 20 \mathrm{pg} / \mathrm{mL}$ and a serum FSH level of $\geq 50 \mathrm{U} / \mathrm{L}$. Women were ineligible for inclusion if they had contraindications to hormone therapy; were receiving anticoagulant therapy; used oral, transdermal or transvaginal hormone therapies within six weeks prior to study entry; used long-acting injections or implants of hormone therapies within six months prior to study entry; had a past medical history significant for diabetes, cardiovascular disease, depression, hypertension, thromboembolism, alcohol or other drug abuse, other condition that could affect study participation (determined by investigator), participation in another clinical study within the previous month or use of an investigational drug within the prior three months.

The primary outcome of this study was the change in the frequency and intensity of hot flushes from baseline. Secondary outcomes included evaluation of other menopausal symptoms such as sweating, insomnia, depression, nervousness and urogenital symptoms as well as vaginal bleeding and other adverse effects. Hot flushes were recorded on patient diary cards in the same manner as that in the Lee et al. [8] study.

By weeks 3 to 16, treatment with estradiol and all doses of drospirenone were associated with a larger decline in hot flush frequency compared to placebo, ranging from $37.5 \%$ to $41.0 \%$ (95\% CI $-25.1 \%$ to $-53.7 \%, p \leq 0.001$ ). By week 16 , the weekly number of moderate to severe hot flushes in women receiving hormone therapy had declined from 21.8 to 29.8 at baseline to 1.0 to 2.0. Weekly mild hot flushes declined from 7.4 to 9.6 at baseline to 1.4 to 3.7 by week 16 . Estradiol/drospirenone was also found to reduce all other menopausal and urogenital symptoms. The most common adverse effects associated with hormone therapy were breast pain and headache. One serious adverse event occurred in a patient receiving estradiol/drospirenone $2 \mathrm{mg}$ which was permanent vaginal bleeding resolved by a hysterectomy and ultimately determined to be adenomyosis uteri interna and several leiomyomata.

The investigators concluded combination therapy of estradiol and drospirenone is effective at reducing the frequency of hot flushes and other menopausal symptoms. Although the investigators had a larger study population, 163 of the 225 (72.4\%) participants were included in the valid-case population as compared to the intent-to-treat population. Additionally, no $p$ values were provided on baseline characteristics, which lends question to the homogeneity of the groups. Lastly, external validity is limited as this was a healthy group of women, and no conclusions can be made about women with any existing conditions upon which they excluded [9]. 
Gambacciani et al. [10] conducted a single-center, prospective, randomized, calcium-controlled study assessing the effects on quality of life of $17 \beta$-estradiol $1 \mathrm{mg} /$ drospirenone $2 \mathrm{mg}$ once daily for three months in postmenopausal Caucasian women. Seventy women aged 60 or younger recruited from menopause clinics in Italy were randomized to receive estradiol/drospirenone $(n=35)$ or calcium $1,000 \mathrm{mg} /$ day $(\mathrm{n}=35)$. Participants were eligible for study entry if they were ammenorhic for at least 1 year before the study, had a FSH level of $>40 \mathrm{IU} / \mathrm{L}$ and estradiol level $<25 \mathrm{pg} / \mathrm{mL}$, and had a systolic blood pressure (SBP) $<140 \mathrm{mmHg}$ and diastolic blood pressure (DBP) $<90 \mathrm{mmHg}$. Patients were excluded from this study if they reported previous intolerance to hormone replacement therapy; did not have vasomotor symptoms; history of hypertension or received antihypertensives within the previous three months; diabetes or other metabolic disorders including dyslipidemia; history of cardiovascular disease; venous thromboembolism or other hemostatic disorder predisposing them to thromboembolic complications; abnormal uterine bleeding; any unstable medical condition; history of alcoholism, drug abuse, psychosis or other emotional/intellectual problems that could impact compliance or informed consent. Though not explicitly stated as criteria for exclusion, none of the women had been treated with hormone-containing preparations in the year prior to study entry.

The investigators did not state what the primary outcome(s) of this study were; however, they stated their aim was to evaluate the quality of life as measured by the Women's Health Questionnaire (WHQ) as well as changes in body mass index (BMI), waist circumference (WC), blood pressure, and insulin values. Of the 70 participants who were randomized, 20 patients receiving calcium and 32 patients receiving hormone therapy were able to be evaluated. This represents a $42.9 \%$ drop out rate for the calcium group and an $8.6 \%$ drop out rate for those receiving treatment. The high rate for the calcium group was explained as being lost to follow up or seeking treatment for climacteric symptoms. For women receiving estradiol/drospirenone, significant reductions from baseline were evident in 6 of the 9 domains of the WHQ, including vasomotor symptoms, sexual behavior, depressed mood, somatic symptoms, anxiety/fear, and sleep problems $(p<0.05)$. Additionally, these were all statistically significant when compared to the control group $(p<0.05)$. There were no significant findings related to menstrual symptoms, memory/concentration and attractiveness with hormone treatment compared to baseline or controls. BMI did not change in either group. WC decreased from $77.8 \pm 1.2 \mathrm{~cm}$ at baseline to $75.2 \pm 1.0 \mathrm{~cm}$ after 3 months with estradiol/drospirenone $(p<0.001)$. While blood glucose levels did not significantly change in those receiving hormone therapy, blood insulin levels decreased from $8.6 \pm 0.7 \mu \mathrm{U} / \mathrm{mL}$ at baseline to $7.5 \pm 0.6 \mu \mathrm{U} / \mathrm{mL}$ after 3 months $(p<0.004)$. A statistically significant reduction in insulin resistance as measured by the homeostasis model assessment (HOMA) was noted with hormone therapy. SBP also significantly decreased afterthree3 months in patients receiving active treatment from baseline $(125.9 \pm 1.8 \mathrm{mmHg}$ to $121.8 \pm 1.4 \mathrm{mmHg})$. When evaluating patients with a $\mathrm{SBP}<130 \mathrm{mmHg}$ at baseline, there were no significant changes. Significant changes, however, were noted in those patients with a baseline SBP 130-139 mmHg. DBP did not significantly change.

The authors concluded that combination therapy with estradiol and drospirenone improved vasomotor symptoms and other quality of life measures as well as cardiovascular risk factors. Findings must be interpreted cautiously given some of the limitations. The data that was evaluated came from a small group of participants (i.e., 52 patients). Additionally, this study was not placebo-controlled. While calcium is important for aging women, participants might have experienced adverse effects such as constipation, which might have lead patients to realize they were not receiving active treatment. 
The patients in this study were recruited from a menopause clinic, which introduces bias. Including patients who seek medical care from gynecology offices or general practitioners did not occur, and this could have increased the population size. Because the exclusion criteria were so extensive and only allowed for healthy women, the efficacy of estradiol and drospirenone for women with any health condition cannot be determined from this study. The investigators did not explicitly state their primary and secondary outcomes for this study. Additionally, $p$ values were not provided for baseline characteristics; therefore, it must be assumed the two groups were homogenous at baseline. There was a large drop-out rate for the control group, which lends question if power was achieved, as it was not discussed. While the authors comment the tool used to assess quality of life (WHQ) has been validated in a large Italian population, it is common for menopausal studies assessing vasomotor and other climacteric symptoms to use patient diaries, which did not occur in this study [10].

Chaikittisilpa et al. [11] conducted a multicenter, open-label, noncomparative, phase 4 study evaluating the efficacy and tolerability of estradiol $1 \mathrm{mg} /$ drospirenone $2 \mathrm{mg}$ as hormone replacement therapy in a population of postmenopausal Thai women experiencing vasomotor and other postmenopausal symptoms. The study was conducted in five centers in Thailand and was limited to postmenopausal women who were at least 45 years old and experienced at least 24 hot flushes of any severity over seven consecutive days during a 14 day screening period. Exclusion criteria included but were not limited to myocardial infarction less than 6 months prior to study entry, severe coronary heart disease, congestive heart failure, uncontrolled hypertension, history of stroke or transient ischemic attacks, thromboembolic disorder, uncontrolled thyroid disorders, insulin dependent diabetes mellitus, and a history of depression of unknown cause. The study consisted of a 12-week treatment phase with a two week follow-up phase in which patients continued to record the number and intensity of hot flushes as well as vaginal bleeding patterns on diary cards. The primary endpoint was the change in frequency of hot flushes from baseline to the end of the 12 week treatment period, which was divided into three groups: women with any kind of reduction in the number of hot flushes, a $90 \%$ reduction, or a $100 \%$ reduction. Neither power nor the types of statistical tests used were given, however statistical significance was set a priori at 0.05 .

A total of 55 patients were enrolled in the study and included in the full analysis set which included any patient who had taken at least one dose of the study medication and had at least one observation after the treatment period had started. At 12 weeks, the authors report that 94.6\% (95\% CI 83.9-98.9) of patients had a reduction in the frequency of hot flushes compared to baseline. In $60 \%$ of the patients, frequency of hot flushes decreased to $10 \%$ or less, and $49.1 \%$ of the patients reported no remaining hot flushes at the end of the treatment period. The mean number of hot flushes decreased from 51.1 at baseline to 13.2 at week 12. The relative reduction in mean number of hot flushes at week 12 was $74.5 \%$ (SD $52.1 \%$ ).

The authors concluded that the combination of estradiol $1 \mathrm{mg}$ plus drospirenone $2 \mathrm{mg}$ was effective in reducing the incidence and severity of hot flushes in postmenopausal Thai women. They also state, "the mild blood pressure lowering effect distinguishes this combination hormone replacement therapy from others, thereby providing additional benefits to patients", however this was not a primary or secondary endpoint and no data regarding the interventions effects on blood pressure was reported. The most frequently reported adverse events were vaginal bleeding or spotting $(54.5 \%)$ and breast tenderness $(25.5 \%)$. 
This study had several limitations including the absence of both power and $p$ values as well as no statistical tests appear to have been utilized. Reported baseline characteristics were limited. It is unknown whether confounders such as medication compliance, and lifestyle factors such as diet, exercise, smoking, and herbal or other medication use which may have an effect on the frequency and severity of hot flushes were assessed. The small and homogenous sample of only Thai women, short duration, and lack of a comparator group make it difficult to extrapolate the findings to a general population of female patients experiencing hot flushes [11].

Lin et al. [12] conducted a double-blind, randomized, placebo controlled, phase III study to determine the efficacy, safety, and tolerability of combined drospirenone $2 \mathrm{mg}$ plus estradiol $1 \mathrm{mg}$ in postmenopausal Chinese women experiencing moderate to severe vasomotor symptoms. The study took place across nine centers in China and inclusion was limited to females 45-65 years old with moderate to severe vasomotor symptoms who had taken no hormonal therapy for at least 4 weeks preceding trial enrollment. Exclusion criteria were extensive and included, but were not limited to, a history of cardiovascular disease, thyroid disorders, clinical depression, malignant or premalignant disease, abnormal glucose tolerance, severe or congenital hypertriglyceridemia, current smoking of more than 15 cigarettes/day, concurrent therapy with prescription medications, and use of herbal or other medication for climacteric disorders. The primary efficacy outcome was the relative change in the number of hot flushes per week in the drospirenone $2 \mathrm{mg}$ plus estradiol $1 \mathrm{mg}$ group verses placebo. Co-primary efficacy variables were the absolute changes in the severity of moderate to severe hot flushes and in the severity of all hot flushes from baseline to the mean values for weeks 3-16; however, the study was not powered to assess these variables. Hot flushes were reported using patient diary cards and classified according to severity using a scale of 0 to 3 . A sample size of 36 women per group was required to obtain $90 \%$ power. All tests were one-sided with a significance level of 0.025 , except for the co-primary efficacy variables, which were analyzed via two-sided rank-sum testing.

A total of 183 patients in the drospirenone $2 \mathrm{mg}$ plus estradiol $1 \mathrm{mg}$ group and 61 in the placebo group were included in the full analysis set which included all randomized patients who took at least one tablet and provided at least one subsequent assessment. Of note, the mean number of hot flushes per week was significantly higher at baseline in the drospirenone $2 \mathrm{mg}$ plus estradiol $1 \mathrm{mg}$ group $(57.8 \pm 36.9)$ than in the placebo group $(50.3 \pm 31.1 ; p=0.0263)$. Per the authors, all other baseline characteristics were similar; however, no other $p$ values were reported. The relative decrease in the mean number of hot flushes per week in the drospirenone $2 \mathrm{mg}$ plus estradiol $1 \mathrm{mg}$ group was $80.4 \%$ and $51.9 \%$ in the placebo group $(p<0.0001)$. The mean number of hot flushes per week was $11.1 \pm 15.1$ in the drospirenone $2 \mathrm{mg}$ plus estradiol $1 \mathrm{mg}$ group and $22.4 \pm 17.3$ in the placebo group, beginning at week 3 of treatment and continuing through week 16. The mean absolute decreases as compared to baseline were $45.9 \pm 29.3$ for the drospirenone $2 \mathrm{mg}$ plus estradiol $1 \mathrm{mg}$ group $(p<0.001)$ and $27.5 \pm 28.1$ for the placebo group $(p<0.001)$. The author's concluded that the study demonstrated the efficacy of drospirenone $2 \mathrm{mg}$ plus estradiol $1 \mathrm{mg}$ for reducing the frequency and severity of VMS in Chinese postmenopausal women.

Even though the study met power and used appropriate statistics, it had a very small sample size and short duration. While the authors did take into account other medications and herbal supplements, they do not appear to have assessed diet and exercise. Furthermore, since this study involved only Chinese women, it is difficult to extrapolate the results to other ethnic groups [12]. 
Archer et al. [13] conducted a multicenter, double-blind, randomized, parallel-group study on the effects of differing doses of drospirenone in combination with estradiol $1 \mathrm{mg}$ versus monotherapy with estradiol $1 \mathrm{mg}$ on endometrial proliferation in postmenopausal women with or without vasomotor and other menopausal symptoms. The primary endpoint in this study was the occurrence of endometrial hyperplasia; however, secondary endpoints included, but were not limited to, hot flush frequency and severity. The number and severity of hot flushes were recorded on diary cards throughout the 1 year duration of the study. Inclusion in the study was limited to women between the ages of 42 and 75 with an intact uterus and normal endometrial histology at baseline. Exclusion criteria included, but were not limited to, abnormal or suspicion of abnormal endometrial histology, myocardial infarction within the last 6 months, heart disease severe enough to have required antiarrhythmic or antianginal medications, thromboembolic disorders within the last three years, history of stroke or transient ischemic attack, as well as known or suspected malignant or premalignant disease of any kind. Participants were randomized to one of five groups of estradiol $1 \mathrm{mg}$ monotherapy or estradiol $1 \mathrm{mg}$ in combination with drospirenone $0.5 \mathrm{mg}, 1 \mathrm{mg}, 2 \mathrm{mg}$, or $3 \mathrm{mg}$.

One thousand one hundred forty-two women received at least one dose of study medication and were included in the intention-to-treat analysis; 845 patients completed the study. The primary endpoint was analyzed using binomial proportion and complementary survival probability. Frequency and severity of hot flushes were converted to interval/ratio data and analyzed via ANOVA. Within group changes from baseline were examined via a two-tailed paired $t$ test for significance and between group differences were tested at a $1.25 \%$ level of significance using the Bonferroni method.

For the primary endpoint, the probability of hyperplasia was 0.060 (95\% CI, 0.043-0.078) for the estradiol monotherapy group. For the drospirenone $2 \mathrm{mg}$ /estradiol $1 \mathrm{mg}$ group the probability of hyperplasia was 0.007 , and for the remaining combination groups the authors state the probability was nonsignificant. In regards to hot flushes, a statistically significant decrease in hot flushes from baseline was seen at all time points beginning at week 2 ( $p \leq 0.008$ for all treatment groups). No statistically significant difference in the mean number of weekly hot flushes was seen between groups. The authors concluded that drospirenone at all studied doses in combination with estradiol was effective in protecting against endometrial hyperplasia and effective treatment for the symptoms associated with menopause, including moderate to severe hot flushes. However, experiencing hot flushes was not an inclusion criterion and only a decrease in the number of hot flushes was reported, so it would be difficult to extrapolate that combination therapy was effective specifically for the treatment of moderate to severe hot flushes. Furthermore, hot flush frequency and severity was a secondary endpoint of this study. Several other limitations include the inclusion of smokers and lack of assessment of diet, exercise, or the use of herbal supplements or medications that may have an effect on menopausal symptoms. The lack of power in this study and the transformation of the data regarding number and severity of hot flushes from nominal and ordinal data to interval ratio are also limitations [13].

\section{Results and Discussion}

A review of the current literature suggests that drospirenone in combination with estrogen is effective at minimizing hot flushes associated with menopause. Six clinical trials were reviewed, four of which explicitly assessed hot flushes as a primary outcome [8-13]. Overall, efficacy ranged 
from modest to large (i.e., $37.5 \%$ to $94.6 \%$ ). The study investigating hot flushes as a secondary outcome found a significant reduction in hot flushes as compared to baseline, but not between treatment groups [13].

The findings from these studies should be interpreted cautiously. While many of these studies were conducted in various countries utilizing participants from several ethnic groups including Korean, Italian, Chinese, American, and Thai women, amongst others, the exclusion criteria was rather extensive and focused on mostly healthy women, thereby reducing the external validity of the findings. Additionally, 2 of the 6 trials did not utilize diary cards for assessing hot flushes [10,13]. Therefore, outcomes were not equally assessed across these 6 trials. The findings from the study assessing hot flushes as a secondary outcome are generally viewed as "hypothesis generating" as the study was not designed and adequately powered to assess this outcome [13]. The number of study participants varied from 52 to 1,142 , with the latter number indicating participants in the study with hot flushes as a secondary outcome [8-13]. When excluding that study and considering only the studies with hot flushes as a primary outcome as well as including one that did not specify if hot flushes was the primary outcome, the largest number of study participants was 244 [12]. All studies were of short duration, 16 weeks or less, except for Archer et al. which had a duration of 1 year [13]. While the duration was long enough to see an effect, it is difficult to know the long term effects of estradiol/drospirenone from these studies alone. Additionally, several of the studies did not provide $p$ values for baseline characteristics, which is essential for assessing the homogeneity of the treatment groups.

Given the findings from the medical literature, it appears that drospirenone-containing HRT is an effective option for healthy women for the treatment of hot flushes associated with menopause. It is unclear if drospirenone-containing hormone replacement therapy confers additional benefits for treating vasomotor symptoms as compared to estradiol alone or in combination with other progestins. Adequate patient assessment is necessary to ensure the appropriate prescribing of this therapy to women without a significant history of estrogen-sensitive cancers or active VTE. Even though patients in these studies did not experience any venous thromboembolic events based on the data provided, judicious and cautious use of drospirenone-containing HRT is warranted in menopausal women given an elevated baseline risk for VTE associated with aging. A review of the literature regarding a VTE risk associated with drospirenone is conflicting. Data from the European Active Surveillance study including new users of oral contraceptives or those switching oral contraceptives found drosperinone-containing oral contraceptives to carry the a similar risk of VTE as compared to other oral contraceptives [14]. On the contrary, data from Lidegaard evaluating the risk of VTE in various estrogen/progestin oral contraceptives found drospirenone-containing oral contraceptives to have at least two times the risk of VTE as compared to oral contraceptives containing levonorgestrel [15]. While there are differences in doses of drospirenone between these populations (i.e., $~ 0.25-0.5 \mathrm{mg}$ of drospirenone in HRT $v s . \sim 3 \mathrm{mg}$ of drospirenone in oral contraceptives), this data serves as a reminder to consider the possibility of VTE when recommending drospirenone-containing HRT.

Given the concern for hyperkalemia with drospirenone, patients taking medications that may raise potassium levels should be identified for monitoring, and those with adrenal insufficiency, hepatic impairment, or renal insufficiency should not receive therapy with drospirenone. In addition, proper patient counseling is warranted to help patients identify signs and symptoms of VTE and what to do should they occur. 


\section{Conclusions}

The available data supports the use of drospirenone-containing HRT for the treatment of hot flushes associated with menopause.

\section{Conflicts of Interest}

The authors declare no conflict of interest.

\section{References}

1. Utian, W.H. Psychosocial and socioeconomic burden of vasomotor symptoms in menopause: A comprehensive review. Health Qual. Life Outcomes 2005, 3, doi:10.1186/1477-7525-3-47.

2. Sturdee, D.W.; Panay, N. Recommendation for the management of postmenopausal vaginal atrophy. Climacteric 2010, 13, 509-522.

3. Pines, A.; Sturdee, D.W.; Birkhäuser, M.H.; Schneider, H.P.; Gambacciani, M.; Panay, N. IMS updated recommendations on postmenopausal hormone therapy. Climacteric 2007, 10, 181-194.

4. Carranza-Lira, S. Safety, efficacy and patient acceptability of drospiranone and estradiol in the treatment of menopausal vasomotor symptoms: A review. Clin. Interv. Aging 2009, 4, 59-62.

5. Yikorkala, O. Drospirenone, a progestin with a unique cardiovascular profile, for safe contraception and treatment of menopausal symptoms. Climacteric 2005, 8, 1-3.

6. Oelkers, W. Drospirenone, a progestogen with antimieralocorticoid properties: A short review. Mol. Cell. Endocrinol. 2004, 217, 255-261.

7. Angeliq ${ }^{\circledR}$ (package insert). Available online: http://berlex.bayerhealthcare.com/html/products/ pi/fhc/Angeliq_PI.pdf(accessed on 28 June 2013).

8. Lee, B.S.; Kang, B.M.; Yoon, B.K.; Choi, H.; Park, H.M.; Kim, J.G. Efficacy and tolerability of estradiol $1 \mathrm{mg}$ and drospirenone $2 \mathrm{mg}$ in postmenopausal Korean women: A double-blind, randomized, placebo-controlled, multicenter study. Maturitas 2007, 57, 361-369.

9. Schurmann, R.; Holler, T.; Benda, N. Estradiol and drospirenone for climacteric symptoms in postmenopausal women: A double-blind, randomized, placebo-controlled study of the safety and efficacy of three dose regimens. Climacteric 2004, 7, 189-196.

10. Gambacciani, M.; Rosano, G.; Cappagli, B.; Pepe, A.; Vitale, C.; Genazzani, A.R. Clinical and metabolic effects of drospirenone-estradiol in menopausal women: A prospective study. Climacteric 2011, 14, 18-24.

11. Chaikittisilpa, S.; Angsuwathana, S.; Chaovisitsaree, S.; Jirapinyo, M.; Wilawan, K.; Panyakhamlerd, K.; Jaisamrarn, U.; Techatraisak, K.; Morakote, N.; Suchartwatnachai, C.; et al. Clinical responses to the combination of estradiol and drospirenone in symptomatic postmenopausal Thai women. J. Med. Assoc. Thai. 2011, 94, 1019-1025.

12. Lin, S.Q.; Sun, L.Z.; Lin, J.F.; Yang, X.; Zhang, L.J.; Qiao, J.; Wang, Z.H.; Xu, Y.X.; Xiong, Z.A.; Zhou, Y.Z.; et al. Estradiol $1 \mathrm{mg}$ and drospirenone $2 \mathrm{mg}$ as hormone replacement therapy in postmenopausal Chinese women. Climacteric 2011, 14, 472-481. 
13. Archer, D.F.; Thorneycroft, I.H.; Foegh, M.; Hanes, V.; Glant, M.D.; Bitterman, P.; Kempson, R.L. Long-term safety of drospirenone-estradiol for hormone therapy: A randomized, double-blind, multicenter trial. Menopause 2005, 12, 716-727.

14. Dinger, J.C.; Heinemann, L.A.J.; Kuhl-Habich, D. The safety of a drospirenone-containing oral contraceptive: Final results from the European Active Surveillance study on oral contraceptives based on 142, 475 women-years of observation. Contraception 2007, 75, 344-354.

15. Lidegaard, O.; Nielsen, L.H.; Skovlund, C.W.; Skjeldestad, F.E.; Lokkegaard, E. Risk of venous thromboembolism from use of oral contraceptives containing different progestogens and oestrogen doses: Danish cohort study, 2001-9. Br. Med. J. 2011, doi:10.1136/bmj.d6423.

(C) 2013 by the authors; licensee MDPI, Basel, Switzerland. This article is an open access article distributed under the terms and conditions of the Creative Commons Attribution license (http://creativecommons.org/licenses/by/3.0/). 